\begin{tabular}{|c|c|c|}
\hline & Int.J.Curr.Microbiol.App.Sci (2021) 10(08): 565-577 & \\
\hline & $\begin{array}{l}\text { International Journal of Current Micro6iology and Applied Sciences } \\
\text { ISSN: 2319-7706 Volume } \mathbf{1 0} \text { Number } \mathbf{0 8}(\mathbf{2 0 2 1 )} \\
\text { Journal homepage: http://www.ijcmas.com }\end{array}$ & $\begin{array}{l}50 \\
502\end{array}$ \\
\hline $\begin{array}{l}\text { EXCELLENT } \\
\text { PUBLISHERS }\end{array}$ & & whwe.jicmascom \\
\hline
\end{tabular}

Original Research Article

https://doi.org/10.20546/ijcmas.2021.1008.067

\title{
Effect of Seed Treatment on Seed Quality and Storability in Wheat (Triticum aestivum L.) in North Western Himalayas
}

\author{
Anubhav Thakur*, K. C. Dhiman and Rajesh Kanwar \\ Department of Seed Science and Technology, CSK Himachal Pradesh Krishi Vishvavidyala, \\ Palampur - 176062, India \\ *Corresponding author
}

A B S T R A C T

\begin{tabular}{|l|}
\hline Ke y w or d s \\
$\begin{array}{l}\text { Wheat, seed } \\
\text { treatment, } \\
\text { storability, seed } \\
\text { quality }\end{array}$ \\
\hline Article Info \\
\hline $\begin{array}{l}\text { Accepted: } \\
\text { 25 July } 2021 \\
\text { Available Online: } \\
10 \text { August } 2021\end{array}$ \\
\hline
\end{tabular}

Storage experiment was conducted to study the effect of polymer, fungicides and insecticide on seed quality parameters and storability in wheat. Experimental material consisted of carry over wheat seeds (variety HPW-155) of rabi 2017 - 18. The observations were recorded bimonthly on parameters viz; germination $(\%)$, seedling length $(\mathrm{cm})$, dry weight $(\mathrm{g})$, vigour index - I, vigour - II, speed of germination, field emergence (\%), 100 seed weight (g) for 12 months of storage. All parameters declined with the advancement in storage period. The results showed that seeds treated with polymer + vitavax $200 @ 2$ $\mathrm{g} / \mathrm{kg}$ of seed, recorded higher germination percentage $(95.00 \%)$, seedling length $(17.58 \mathrm{~cm})$, seedling dry weight $(0.0138 \mathrm{~g})$, vigour index - I (1670) \& vigour - II (1.311), speed of germination (19.98), 100 seed weight (5.54 g) and field emergence $(87.33 \%)$ which was at par with vitavax $200 @ 2 \mathrm{~g} / \mathrm{kg}$ of seed, over untreated control $\left(\mathrm{T}_{1}\right)$. So it can be concluded that for maintain seed quality and enhancing storability, seed of wheat can either be treated with polymer@3 ml/kg of seed + vitavax 200 @ g/ kg of seed or vitavax 200 @ 2 $\mathrm{g} / \mathrm{kg}$ of seed.

\section{Introduction}

Quality seed, being the basic input in agriculture, plays an important role in realizing optimum yield and productivity of any crop. Seed with high quality and vigour will not only help in achieving optimum plant population in the field but also result in vigorous seedling, which can surpass the initial abiotic and biotic stresses to significant extent (Jacob et al., 2016). Deterioration of seed during storage is inevitable and leads to different changes at various levels viz., impairment or shift in metabolic activity, compositional changes, decline or change in enzyme activities, phenotypic, cytological changes apart from quantitative losses. Being hygroscopic in nature the viability and vigour 
of seeds under storage are known to be regulated by variations in the physio-chemical factors, initial seed quality, storage structures, packaging materials (Doijode, 2000). The rate of seed deterioration can be slowed down either by storing the seeds under controlled or by treating the seeds with various seed treating chemicals. As the controlled conditions involve huge cost, seed treatment remains the best alternative approach to maintain the seed quality. From the past few decades, seed treatment technology has evolved through research innovations and has delivered several products that can be used to manipulate the performance potential of the seeds and subsequent crop (Jacob et al., 2016). Seed treatment technology is being widely used for different purposes like synchronization of flowering between parental lines (Johnson et al., 1999), for precision sowing (Lagoa et al., 2012), for seed quality enhancement (Kumar et al., 2007), delivery of nutrients (Farooq et al., 2012), for early sowing in cold and wet soils (Sharratt and Gesch, 2008), delivery of growth hormones (Gevrek et al., 2012), delivery of pesticides (Sherry et al., 2007), delivery of bio control agents (Mastouri et al., 2010), as a bioactive coating (Ziani et al., 2010) and as anti-counterfeiting technology for eliminating fake seeds in the market (Guan et al., 2013). Thus, with the seed treatment concept becoming a practical reality for different applications, there is now accumulating interest in multiple aspects of this technology, whereby its application can be extended to different crops that are grown and stored under different agro-climatic conditions. Among these concepts, polymer film coating has been proven to enhance the crop establishment particularly under adverse conditions.

The polymer film coat also provides protection from the stress imposed by accelerated ageing, which includes fungal invasion. The coat is thin $(8 \mu \mathrm{m})$, simple to apply, diffuses rapidly and non-toxic to the seedlings during germination. It improves plant stand and emergence of seeds, helps in accurate application of the chemical, reduces chemical wastage and helps to make room for all required ingredients, protectants, nutrients, plant growth promoters, hydrophobic / hydrophilic substance and oxygen suppliers etc. By encasing the seed within a thin film of biodegradable polymer, the adherence of seed treating chemicals to seed can be improved, ensures dust free handling, making treated seed both useful and environment friendly. Currently seed treating polymer are being used by seed companies along with other active ingredients such as insecticides and fungicides. This help in improving the resistance of seeds towards pest and diseases in the much warranted juvenile stage, besides improves the seedling vigour and ultimately the plant stands and yield. The polymer film also acts as physical barrier, which has been reported to reduce the leaching of inhibitors from the seed coverings and may restrict oxygen diffusion to the embryo. (Vanangamudi et al., 2003).

In north western Himalayas region, the information on storability of carryover wheat seeds is inadequate and the quality of wheat seed deteriorates mainly due to storage of untreated seed, storage conditions and the packaging materials used for storage of seed. So the present study was conducted to study the effect of seed treatment on seed quality and storability in wheat.

\section{Materials and Methods}

The current study was conducted at the Department of Seed Science and Technology, CSKHPKV Palampur. Experimental material consisted of carry over wheat seed (variety HPW -155) of rabi 2017 - 18. The experiment was conducted under a completely randomized design (CRD) and each treatment 
was replicated three times (Table 1). Seeds are stored in high density polyethylene bags (HDPE). The seed quality parameters were evaluated at bimonthly interval for twelve months from December 2018 to December 2019. The seeds were drawn at random from each treatment bag replication - wise. Germination test was conducted using 100 seeds in four replications by adopting blotter paper method as described by ISTA procedures (Anonymous 1999). Seeds were incubated in germinator at a temperature $25 \pm 1^{\circ} \mathrm{C}$ and $\mathrm{RH}$ of 90 percent. Normal germinated seeds were counted on $4^{\text {th }}$ and $8^{\text {th }}$ day after incubation and total germination percentage was calculated using the formula at the end of test period:

Germination $(\%)=$ (Number of normal germinated seeds/ Total number of seeds) $\times 100$

Speed of germination was determined on the basis of daily germination count and was calculated by the following formula.

Speed of germination $=\mathrm{n} 1 / \mathrm{d} 1+\mathrm{n} 2 / \mathrm{d} 2+$ n3/d3+-----

Where,

$\mathrm{n}$ - number of germinated seeds, $\mathrm{d}$ - number of days.

Ten normal seedlings on $8^{\text {th }}$ day of germination test were selected randomly from each replication of the treatment for measuring the seedling length and the average was worked out in centimeters. The same ten normal seedlings were then used for measurement of seedling dry weight. The seedlings were put butter paper bag and placed in hot air oven at $80^{\circ} \mathrm{C}$ for 24 hours. The dry weight of the seedlings was recorded and expressed in grams per seedling. The seedling vigour index - I and vigour index - II were calculated by adopting the method suggested by Abdul Baki and Anderson (1973).

SVI $-\mathrm{I}=$ Germination $(\%) \times$ Seedling length (cm), and

SVI - I = Germination $(\%) \times$ Seedling dry weight $(\mathrm{g})$

Field emergence count was taken on the $14^{\text {th }}$ day after sowing and the emergence percentage was calculated taking into account the number of seedlings emerging above the soil surface. Moisture content of seeds was recorded in percentage using a moisture meter (Non - Destructive PM 600).

The laboratory data was statistically analyzed using software OPSTAT (Sheoran et al., 1998). The data on germination (\%) and field emergence $(\%)$ were transformed into arc sine root percentage and transformed data were used for statistical analysis.

\section{Results and Discussion}

Most of seed quality parameters declined with the advancement of storage period. Mean germination percentage (final count) in the beginning and end of storage period was 96.30 and 92.2., respectively (Table 2). The reduction in seed germination over time could also be due to the reduction in enzyme activity within the seed. Initially significantly highest germination percentage was recorded in $\mathrm{T}_{6}-$ (98.66 \%) which was at par with $\mathrm{T}_{5}$ (98.00\%), followed by $\mathrm{T}_{8}-(96.66 \%)$ which was at par with $\mathrm{T}_{7}-(96.66 \%), \mathrm{T}_{10}-(96.66 \%)$, $\mathrm{T}_{3}$ - flowable thiram (Royal flow $40 \mathrm{SC}$ ) @ $2.4 \mathrm{ml} / \mathrm{kg}(96.00 \%)$, followed by $\mathrm{T}_{2}$ $(95.33 \%)$ which was at par with $\mathrm{T}_{9}-(95.00 \%)$ and $\mathrm{T}_{4}-(95.00 \%)$ as while lowest was recorded in $\mathrm{T}_{1}$ - control $(95.00 \%)$. The same pattern was recorded till six months. At $8^{\text {th }}$ month the trend varied and significantly highest germination (\%) was recorded for $\mathrm{T}_{6}$ - 
$(95.67 \%)$ which was at par with $\mathrm{T}_{5}-(95.00$ $\%)$, followed by $\mathrm{T}_{8}-(94.33 \%)$ compared to $\mathrm{T}_{1}$ - control $(91.00 \%)$. The same pattern was recorded till the end of storage period. At the end of storage, significantly highest germination percentage was recorded for $\mathrm{T}_{6}$ $(95.00 \%)$ which was at par with $\mathrm{T}_{5}-(94.33$ $\%$ ) while lowest was recorded in $\mathrm{T}_{1}$ - control $(89.00 \%)$. The higher germination percentage was recorded in seeds treated with fungicides is due to increase in rate of imbibitions where the fine particles in the treatment act as moisture attracting material to improve germination. The polymer treatment on seeds acts as a physical barrier that has been reported to reduce leaching of inhibitors from the seed covering and may restrict oxygen diffusion to the embryo (Duan and Burris 1997). The results are in accordance with the finding of Parihar et al., (2019) in okra who reported that seed coated with polymer @ 3 $\mathrm{ml} / \mathrm{kg}$ of seed + vitavax $200 @ 2 \mathrm{~g} / \mathrm{kg}$ of seeds showed significantly higher germination over untreated control at the end of one year of storage. Similarly, radish seed treated with polymer@ $3 \mathrm{ml} / \mathrm{kg}$ of seed+ vitavax 200 @ 2 $\mathrm{g} / \mathrm{kg}$ of seed also recorded significantly higher germination percentage over untreated control after 12 months of storage (Kotia 2020).

The speed of germination varied significantly throughout the storage period till the end. Irrespective of different seed treatments, the speed of germination continued to decrease from initial to last month of the storage. On an average, the speed of germination in the beginning and end of the storage period was 20.65 and 18.83, respectively (Table 2). Initially higher speed of germination was recorded in $\mathrm{T}_{6}-(21.42)$ which was at par with $\mathrm{T}_{5}-(21.40)$, followed by $\mathrm{T}_{8}-(20.49)$ which was at par with $\mathrm{T}_{7}-(20.49), \mathrm{T}_{10}-(20.48)$ and $\mathrm{T}_{3}$ - (20.47), followed by $\mathrm{T}_{2}-(20.45)$, which was at par with $\mathrm{T}_{9}-(20.45)$, and $\mathrm{T}_{4}-(20.45)$ as compared to $\mathrm{T}_{1}$ - untreated control (20.45). The same pattern was recorded till six months of storage and after $8^{\text {th }}$ month, significantly higher speed of germination was recorded for $\mathrm{T}_{6}$ - (21.00) which was at par with $\mathrm{T}_{5}$ (20.98), followed by $\mathrm{T}_{8}-(19.95)$ as compared to $\mathrm{T}_{1}$ - control (19.50). The same pattern was recorded till end of storage period and significantly higher speed of germination was recorded in $\mathrm{T}_{6}-(19.98)$ which was at par with $\mathrm{T}_{5}$ - (19.96) over $\mathrm{T}_{1}$ - control (18.10). Speed of germination decreased with the increase in storage period. The reduction in speed of germination of seeds might be due to restricted oxygen supply to the embryo and retention of water soluble germination inhibitors. Similar findings were reported by Padhi et al., (2017) and Sharma et al., (2017) in paddy, who studied the effect of polymer @ $4 \mathrm{ml}+$ vitavax 200 @ $2 \mathrm{~g} / \mathrm{kg}$ of seed and recorded significantly higher speed of germination over untreated control after one year of storage.

Irrespective of different seed treatments, the seedling length $(\mathrm{cm})$ decreased with the advancement in the storage period. Average seedling length recorded at the beginning and the end of storage period was $18.93 \mathrm{~cm}$ and $16.94 \mathrm{~cm}$, respectively (Table 3). The decline in seedling length may be attributed to age induced decline in germination, the damage caused by accumulation of toxic metabolites which might have hindered the seedling growth. Initially significantly highest seedling length was recorded for $\mathrm{T}_{6}-(18.99 \mathrm{~cm})$ which was at par with $\mathrm{T}_{5}-(18.98 \mathrm{~cm})$, followed by $\mathrm{T}_{8}-(18.95 \mathrm{~cm})$ which was at par with $\mathrm{T}_{7}-$ $(18.94 \mathrm{~cm}), \mathrm{T}_{10}-(18.94 \mathrm{~cm}), \mathrm{T}_{3}-(18.92 \mathrm{~cm})$, followed by $\mathrm{T}_{2}-(18.91 \mathrm{~cm})$ which was at par with $\mathrm{T}_{9}-(18.90 \mathrm{~cm})$, and $\mathrm{T}_{4}-(18.90 \mathrm{~cm})$ as compared to $\mathrm{T}_{1}-$ control $(18.90 \mathrm{~cm})$. The similar pattern was recorded till $6^{\text {th }}$ month of storage.

At $8^{\text {th }}$ month the trend varied and significantly highest seedling length was recorded for $\mathrm{T}_{6}$ $(18.32 \mathrm{~cm})$ which was at par with $\mathrm{T}_{5}-(18.30$ 
$\mathrm{cm})$, followed by $\mathrm{T}_{8}-(18.26 \mathrm{~cm}), \mathrm{T}_{7}-(18.22$ $\mathrm{cm})$, while the lowest was recorded in $\mathrm{T}_{1}$ control $(15.12 \mathrm{~cm})$. It may be due to proper supply of water, nutrients and due to induction of photosynthesis and synthesis of alpha amylase, protease and other hydrolytic enzymes which appear to induce the activity of gluconeogenic enzymes during early stage of seed germination causing higher seedling length (Huang et al., 2021). Roopashree et al., (2018) and Parihar et al., (2019) in soybean and okra seeds reported the similar results. They recorded that seeds treated with polymer and vitavax had resulted in significantly higher seedling length as compared to untreated control after 12 months of storage.

Almost similar trends were recorded for seedling dry weight (g). Seedling dry weight decreased from initial to last month of the storage. On an average, dry weight per seedling at the beginning and end of storage period was $0.0167 \mathrm{~g}$ and $0.0125 \mathrm{~g}$, respectively (Table 3). In the initial month of storage, significantly maximum seedling dry weight $(\mathrm{g})$ was recorded for $\mathrm{T}_{6}-(0.0173 \mathrm{~g})$ which was at par with $\mathrm{T}_{5}-(0.0171 \mathrm{~g})$, followed by $\mathrm{T}_{8}-$ $(0.0169 \mathrm{~g})$ which was at par with $\mathrm{T}_{7}-(0.0167$ g), $\mathrm{T}_{10}-(0.0167 \mathrm{~g}), \mathrm{T}_{3}-(0.0167 \mathrm{~g})$, followed by $\mathrm{T}_{2}-(0.0165 \mathrm{~g})$ which was at par with $\mathrm{T}_{9}-$
$(0.0165 \mathrm{~g}), \mathrm{T}_{4}-(0.0165 \mathrm{~g})$ while the lowest was recorded in $\mathrm{T}_{1}-$ control $(0.0164 \mathrm{~g})$. The same pattern was recorded till six months of storage.

At $8^{\text {th }}$ month the trend varied and significantly maximum dry weight $(\mathrm{g})$ was recorded for $\mathrm{T}_{6}$ $(0.0156 \mathrm{~g})$ which was at par with $\mathrm{T}_{5}-(0.0154$ $\mathrm{g})$, followed by $\mathrm{T}_{8}-(0.0152 \mathrm{~g}), \mathrm{T}_{7}-(0.151 \mathrm{~g})$ as compared to $\mathrm{T}_{1}-$ control $(0.0134 \mathrm{~g})$. The same pattern was recorded till end of storage period. At the end of storage, significantly maximum dry weight $(\mathrm{g})$ was recorded for $\mathrm{T}_{6}$ $(0.0138 \mathrm{~g})$ which was at par with $\mathrm{T}_{5}-(0.0136$ g) while the lowest was recorded in $\mathrm{T}_{1}$ control $(0.0109 \mathrm{~g})$. The dry matter production of seedling is the ultimate manifestation of physiological vigour. This is a physiological phenomenon influenced by reserve metabolites, enzyme activities and growth regulators (Geetha and Bhaskharan 2020).

These results are in the conformity with finding of Sharma et al., (2017) who observed significantly higher seedling dry weight in maize seeds treated with polymer @ $3 \mathrm{ml} / \mathrm{kg}$ of seed + vitavax 200@2 g/ $\mathrm{kg}$ of seed over untreated seeds at end of one year of storage period.

Table.1 Details of different seed treatment employed in study

\begin{tabular}{|c|c|}
\hline $\mathbf{T}_{1}$ & Control (Untreated seeds) \\
\hline $\mathbf{T}_{2}$ & Polymer coating (Polykote @ $3 \mathrm{ml} / \mathrm{kg}$ ) of seed, diluted with $5 \mathrm{ml}$ of water) \\
\hline $\mathbf{T}_{3}$ & Flowable thiram (Royal flow 40SC) @ $2.4 \mathrm{ml} / \mathrm{kg}$ \\
\hline $\mathbf{T}_{4}$ & Polymer + flowable thiram (Royal flow 40 SC) @ 2.4 ml $/ \mathrm{kg}$ seed \\
\hline $\mathbf{T}_{5}$ & Vitavax 200 (containing thiram 37.5\% and carboxil 37.5\%)@2 g/kg seed \\
\hline $\mathbf{T}_{6}$ & Polymer + vitavax 200 (containing thiram, 37.5\% and carboxil, 37.5\%) @ $2 \mathrm{~g} / \mathrm{kg}$ of seed \\
\hline $\mathbf{T}_{7}$ & Imidacloprid (Gaucho) @ 4 ml/kg seed \\
\hline $\mathbf{T}_{8}$ & Polymer + flowable thiram (Gaucho) @ 4 ml/kg seed \\
\hline $\mathbf{T}_{9}$ & Polymer + flowable thiram (Royal flow 40 SC) @ 2.4 ml $/ \mathrm{kg} \mathrm{seed} \mathrm{+} \mathrm{imidacloprid} \mathrm{(Gaucho)} \mathrm{@} 4$ ml $/ \mathrm{kg}$ seed \\
\hline $\mathbf{T}_{10}$ & Polymer + vitavax 200 (containing thiram, 37.5\% and carboxil, 37.5\%)@2 g/kg of seed + imidacloprid (Gaucho) @ $4 \mathrm{ml} / \mathrm{kg}$ seed. \\
\hline
\end{tabular}


Table.2 Effect of different seed treatment on germination (\%) and speed of germination

\begin{tabular}{|c|c|c|c|c|c|c|c|c|c|c|c|c|c|c|}
\hline \multirow{3}{*}{ Treatments } & \multicolumn{14}{|c|}{ Months after storage } \\
\hline & \multicolumn{7}{|c|}{ Germination (\%) } & \multicolumn{7}{|c|}{ Speed of germination } \\
\hline & Initial & 2 & 4 & 6 & 8 & 10 & 12 & Initial & 2 & 4 & 6 & 8 & 10 & 12 \\
\hline \multirow[t]{2}{*}{$\mathbf{T}_{1}$} & 95.00 & 93.66 & 93.00 & 93.00 & 91.00 & 90.00 & 89.00 & \multirow[t]{2}{*}{20.45} & \multirow[t]{2}{*}{20.40} & \multirow[t]{2}{*}{20.34} & \multirow[t]{2}{*}{20.20} & \multirow[t]{2}{*}{19.50} & \multirow[t]{2}{*}{19.00} & \multirow[t]{2}{*}{18.10} \\
\hline & (77.04) & $(75.40)$ & (74.63) & $(74.63)$ & $(72.51)$ & (71.54) & $(70.60)$ & & & & & & & \\
\hline \multirow[t]{2}{*}{$\mathbf{T}_{2}$} & 95.33 & 94.33 & 93.66 & 93.33 & 92.33 & 91.66 & 91.33 & \multirow[t]{2}{*}{20.45} & \multirow[t]{2}{*}{20.42} & \multirow[t]{2}{*}{20.35} & \multirow[t]{2}{*}{20.22} & \multirow[t]{2}{*}{19.85} & \multirow[t]{2}{*}{19.2} & \multirow[t]{2}{*}{18.43} \\
\hline & $(77.50)$ & $(76.21)$ & $(75.40)$ & $(75.02)$ & $(73.90)$ & $(73.20)$ & $(72.86)$ & & & & & & & \\
\hline \multirow[t]{2}{*}{$\mathbf{T}_{3}$} & 96.00 & 94.66 & 94.33 & 94.00 & 93.33 & 92.66 & 92.00 & \multirow[t]{2}{*}{20.47} & \multirow[t]{2}{*}{20.43} & \multirow[t]{2}{*}{20.37} & \multirow[t]{2}{*}{20.23} & \multirow[t]{2}{*}{19.87} & \multirow[t]{2}{*}{19.4} & \multirow[t]{2}{*}{18.50} \\
\hline & $(78.43)$ & $(76.62)$ & $(76.21)$ & $(75.79)$ & $(75.02)$ & $(74.27)$ & (73.54) & & & & & & & \\
\hline $\mathbf{T}_{4}$ & 95.00 & 94.00 & 93.33 & 93.00 & 92.00 & 91.33 & 91.00 & 20.45 & 20.41 & 20.35 & 20.21 & 19.83 & 19.18 & 18.41 \\
\hline & (77.04) & (75.79) & $(75.02)$ & $(74.63)$ & $(73.54)$ & (72.86) & $(72.51)$ & & & & & & & \\
\hline $\mathbf{T}_{5}$ & 98.00 & 96.00 & 96.00 & 95.66 & 95.00 & 94.33 & 94.33 & 21.4 & 21.33 & 21.23 & 21.13 & 20.98 & 20.48 & 19.96 \\
\hline & $(81.83)$ & (78.49) & (78.43) & (77.97) & (77.04) & $(76.21)$ & $(76.21)$ & & & & & & & \\
\hline$T_{6}$ & 98.66 & 97.00 & 97.00 & 96.66 & 95.67 & 95.00 & 95.00 & 21.42 & 21.35 & 21.25 & 21.15 & 21.00 & 20.5 & 19.98 \\
\hline & (83.43) & (79.99) & (79.99) & (79.47) & (77.97) & (77.05) & (77.05) & & & & & & & \\
\hline $\mathbf{T}_{7}$ & 96.66 & 95.33 & 95.00 & 94.33 & 94.00 & 93.33 & 93.00 & 20.49 & 20.44 & 20.38 & 20.25 & 19.93 & 19.58 & 18.93 \\
\hline & (79.47) & $(77.51)$ & (77.05) & $(76.21)$ & (75.79) & $(75.02)$ & (74.63) & & & & & & & \\
\hline $\mathbf{T}_{8}$ & 96.66 & 95.66 & 95.33 & 95.00 & 94.33 & 93.66 & 93.33 & 20.49 & 20.45 & 20.39 & 20.26 & 19.95 & 19.6 & 18.95 \\
\hline & (79.47) & (76.97) & (77.51) & (77.04) & $(76.21)$ & $(75.40)$ & (75.02) & & & & & & & \\
\hline $\mathbf{T}_{9}$ & 95.00 & 94.33 & 93.33 & 93.33 & 92.00 & 91.66 & 91.33 & 20.45 & 20.41 & 20.35 & 20.21 & 19.84 & 19.19 & 18.42 \\
\hline & (77.04) & $(76.21)$ & (75.02) & $(75.02)$ & (73.54) & $(73.20)$ & (72.86) & & & & & & & \\
\hline $\mathbf{T}_{10}$ & 96.66 & 95.33 & 94.66 & 94.33 & 93.33 & 92.67 & 92.33 & 20.48 & 20.43 & 20.38 & 20.24 & 19.90 & 19.44 & 18.6 \\
\hline & (79.47) & $(77.51)$ & (76.63) & $(76.21)$ & $(75.02)$ & (74.27) & (73.90) & & & & & & & \\
\hline Mean & 96.30 & 95.03 & 94.56 & 94.26 & 93.30 & 92.63 & 92.27 & 20.65 & 20.60 & 20.54 & 20.41 & 20.06 & 19.56 & 18.83 \\
\hline & (78.87) & (77.08) & (76.48) & (76.10) & (74.96) & $(74.21)$ & (73.82) & & & & & & & \\
\hline SE $(\mathbf{m} \pm)$ & 0.408 & 0.415 & 0.445 & 0.337 & 0.286 & 0.33 & 0.263 & 0.008 & 0.007 & 0.01 & 0.012 & 0.008 & 0.007 & 0.007 \\
\hline CD $(5 \%)$ & 1.21 & 1.23 & 1.33 & 1 & 0.85 & 0.98 & 0.78 & 0.023 & 0.022 & 0.031 & 0.035 & 0.022 & 0.021 & 0.02 \\
\hline
\end{tabular}

Figure in parentheses indicated arc sine value 
Table.3 Effect of different seed treatments on seedling length $(\mathrm{cm})$ and seedling dry weight $(\mathrm{g})$ in wheat during storage

\begin{tabular}{|c|c|c|c|c|c|c|c|c|c|c|c|c|c|c|}
\hline \multicolumn{15}{|c|}{ Months after storage } \\
\hline Treatments & \multicolumn{7}{|c|}{ Seedling length $(\mathrm{cm})$} & \multicolumn{7}{|c|}{ Seedling dry weight (g) } \\
\hline & Initial & 2 & 4 & 6 & 8 & 10 & 12 & Initial & 2 & 4 & 6 & 8 & 10 & 12 \\
\hline $\mathbf{T}_{1}$ & 18.90 & 18.87 & 18.84 & 18.74 & 17.60 & 16.90 & 15.12 & 0.0164 & 0.0162 & 0.0156 & 0.0154 & 0.0134 & 0.0123 & 0.0109 \\
\hline $\mathbf{T}_{3}$ & 18.92 & 18.89 & 18.86 & 18.77 & 17.95 & 17.63 & 16.93 & 0.0167 & 0.0164 & 0.0160 & 0.0158 & 0.0145 & 0.0135 & 0.0124 \\
\hline $\mathbf{T}_{4}$ & 18.90 & 18.87 & 18.84 & 18.74 & 17.85 & 17.53 & 16.83 & 0.0165 & 0.0163 & 0.0156 & 0.0154 & 0.0141 & 0.0129 & 0.0118 \\
\hline $\mathbf{T}_{5}$ & 18.98 & 18.94 & 18.90 & 18.84 & 18.30 & 17.90 & 17.55 & 0.0171 & 0.0170 & 0.0167 & 0.0163 & 0.0154 & 0.0146 & 0.0136 \\
\hline $\mathbf{T}_{7}$ & 18.94 & 18.91 & 18.88 & 18.79 & 18.22 & 17.78 & 17.32 & 0.0167 & 0.0166 & 0.0162 & 0.0160 & 0.0151 & 0.0141 & 0.0130 \\
\hline $\mathbf{T}_{8}$ & 18.95 & 18.91 & 18.89 & 18.80 & 18.26 & 17.82 & 17.37 & 0.0169 & 0.0167 & 0.0164 & 0.0161 & 0.0152 & 0.0143 & 0.0132 \\
\hline $\mathbf{T}_{9}$ & 18.90 & 18.87 & 18.85 & 18.74 & 17.87 & 17.55 & 16.85 & 0.0165 & 0.0163 & 0.0157 & 0.0155 & 0.0142 & 0.0131 & 0.0119 \\
\hline $\mathbf{T}_{10}$ & 18.94 & 18.90 & 18.88 & 18.78 & 18.00 & 17.70 & 17.00 & 0.0167 & 0.0166 & 0.0161 & 0.0159 & 0.0148 & 0.0139 & 0.0127 \\
\hline Mean & 18.93 & 18.90 & 18.87 & 18.78 & 18.02 & 17.63 & 16.94 & 0.0167 & 0.0166 & 0.0161 & 0.0159 & 0.0147 & 0.0137 & 0.0125 \\
\hline SE (m) & 0.010 & 0.009 & 0.010 & 0.011 & 0.016 & 0.021 & 0.022 & 0.0001 & 0.0001 & 0.0001 & 0.0001 & 0.0001 & 0.0001 & 0.0001 \\
\hline
\end{tabular}


Table.4 Effect of different seed treatments on field emergence (\%) in wheat during storage

\begin{tabular}{|c|c|c|c|c|c|c|c|}
\hline \multicolumn{8}{|c|}{ Months after storage } \\
\hline \multirow[t]{2}{*}{ Treatments } & \multicolumn{7}{|c|}{ Field emergence $(\%)$} \\
\hline & Initial & 2 & 4 & 6 & 8 & 10 & 12 \\
\hline $\mathbf{T}_{1}$ & $\begin{array}{c}91.33 \\
(72.85)\end{array}$ & $\begin{array}{c}89.33 \\
(70.91)\end{array}$ & $\begin{array}{c}87.33 \\
(69.12)\end{array}$ & $\begin{array}{c}86.00 \\
(68.03)\end{array}$ & $\begin{array}{c}80.33 \\
(63.65)\end{array}$ & $\begin{array}{c}78.33 \\
(62.33)\end{array}$ & $\begin{array}{c}76.00 \\
(60.64)\end{array}$ \\
\hline $\mathbf{T}_{2}$ & $\begin{array}{c}91.33 \\
(72.85)\end{array}$ & $\begin{array}{c}89.66 \\
(71.22)\end{array}$ & $\begin{array}{c}88.00 \\
(69.71)\end{array}$ & $\begin{array}{c}86.66 \\
(68.55)\end{array}$ & $\begin{array}{c}85.33 \\
(67.45)\end{array}$ & $\begin{array}{c}83.33 \\
(65.88)\end{array}$ & $\begin{array}{c}81.00 \\
(64.13)\end{array}$ \\
\hline $\mathbf{T}_{3}$ & $\begin{array}{c}92.33 \\
(73.90)\end{array}$ & $\begin{array}{c}90.33 \\
(71.86)\end{array}$ & $\begin{array}{c}88.33 \\
(70.00)\end{array}$ & $\begin{array}{c}87.33 \\
(69.12)\end{array}$ & $\begin{array}{c}86.33 \\
(68.27)\end{array}$ & $\begin{array}{c}84.33 \\
(66.65)\end{array}$ & $\begin{array}{c}82.33 \\
(65.12)\end{array}$ \\
\hline $\mathbf{T}_{4}$ & $\begin{array}{c}91.33 \\
(72.85)\end{array}$ & $\begin{array}{c}89.33 \\
(70.91)\end{array}$ & $\begin{array}{c}87.66 \\
(69.41)\end{array}$ & $\begin{array}{c}86.33 \\
(68.27)\end{array}$ & $\begin{array}{c}84.66 \\
(66.92)\end{array}$ & $\begin{array}{c}82.66 \\
(65.37)\end{array}$ & $\begin{array}{r}80.33 \\
(63.65) \\
\end{array}$ \\
\hline $\mathbf{T}_{6}$ & $\begin{array}{c}93.66 \\
(75.40)\end{array}$ & $\begin{array}{c}93.00 \\
(74.62)\end{array}$ & $\begin{array}{c}91.33 \\
(72.85)\end{array}$ & $\begin{array}{c}90.00 \\
(71.55)\end{array}$ & $\begin{array}{c}89.00 \\
(70.60)\end{array}$ & $\begin{array}{c}87.66 \\
(69.41)\end{array}$ & $\begin{array}{r}86.66 \\
(68.55)\end{array}$ \\
\hline $\mathbf{T}_{\mathbf{5}}$ & $\begin{array}{c}94.33 \\
(76.21)\end{array}$ & $\begin{array}{c}94.00 \\
(75.82)\end{array}$ & $\begin{array}{c}93.00 \\
(74.65)\end{array}$ & $\begin{array}{c}91.00 \\
(72.51)\end{array}$ & $\begin{array}{c}89.33 \\
(70.91)\end{array}$ & $\begin{array}{c}88.33 \\
(70.00)\end{array}$ & $\begin{array}{c}87.33 \\
(69.12)\end{array}$ \\
\hline $\mathbf{T}_{7}$ & $\begin{array}{c}92.66 \\
(74.26)\end{array}$ & $\begin{array}{c}91.33 \\
(72.85)\end{array}$ & $\begin{array}{c}89.33 \\
(70.91)\end{array}$ & $\begin{array}{c}88.66 \\
(70.30)\end{array}$ & $\begin{array}{c}87.66 \\
(69.70)\end{array}$ & $\begin{array}{c}86.00 \\
(68.00)\end{array}$ & $\begin{array}{c}84.33 \\
(66.65)\end{array}$ \\
\hline $\mathbf{T}_{8}$ & $\begin{array}{c}92.66 \\
(74.26)\end{array}$ & $\begin{array}{c}91.33 \\
(72.85)\end{array}$ & $\begin{array}{c}89.33 \\
(70.91)\end{array}$ & $\begin{array}{c}89.00 \\
(70.65)\end{array}$ & $\begin{array}{c}88.66 \\
(70.30)\end{array}$ & $\begin{array}{c}86.33 \\
(68.27\end{array}$ & $\begin{array}{c}85.00 \\
(67.18)\end{array}$ \\
\hline $\mathbf{T}_{9}$ & $\begin{array}{c}91.33 \\
(72.85)\end{array}$ & $\begin{array}{c}89.66 \\
(71.22)\end{array}$ & $\begin{array}{c}88.00 \\
(70.00)\end{array}$ & $\begin{array}{c}86.66 \\
(68.55)\end{array}$ & $\begin{array}{c}85.00 \\
(67.18)\end{array}$ & $\begin{array}{c}83.00 \\
(65.62)\end{array}$ & $\begin{array}{c}80.66 \\
(63.89)\end{array}$ \\
\hline $\mathbf{T}_{10}$ & $\begin{array}{c}92.66 \\
(74.26)\end{array}$ & $\begin{array}{c}91.00 \\
(72.53)\end{array}$ & $\begin{array}{c}89.00 \\
(70.61)\end{array}$ & $\begin{array}{c}88.33 \\
(70.00)\end{array}$ & $\begin{array}{c}87.33 \\
(69.12)\end{array}$ & $\begin{array}{c}85.33 \\
(67.45)\end{array}$ & $\begin{array}{c}83.33 \\
(65.88)\end{array}$ \\
\hline Mean & $\begin{array}{c}92.36 \\
(73.92)\end{array}$ & $\begin{array}{c}90.90 \\
(72.41)\end{array}$ & $\begin{array}{c}89.13 \\
(70.72)\end{array}$ & $\begin{array}{c}88.00 \\
(69.70)\end{array}$ & $\begin{array}{c}86.40 \\
(68.33)\end{array}$ & $\begin{array}{c}84.53 \\
(66.81)\end{array}$ & $\begin{array}{c}82.70 \\
(65.39)\end{array}$ \\
\hline $\mathrm{SE}(\mathbf{m})$ & 0.363 & 0.394 & 0.402 & 0.533 & 0.234 & 0.240 & 0.256 \\
\hline $\mathrm{CD}(5 \%)$ & 1.080 & 1.170 & 1.190 & 1.680 & 0.696 & 0.714 & 0.760 \\
\hline
\end{tabular}

Figure in parentheses indicates arc sine value 
Table.5 Effect of different seed treatments on 100 seed weight $(\mathrm{g})$ in wheat during storage

\begin{tabular}{|c|c|c|c|c|c|c|c|}
\hline \multirow[b]{3}{*}{ Treatments } & \multicolumn{7}{|c|}{ Months after storage } \\
\hline & \multicolumn{7}{|c|}{100 seed weight (g) } \\
\hline & Initial & 2 & 4 & 6 & 8 & 10 & 12 \\
\hline $\mathbf{T}_{1}$ & 5.40 & 5.27 & 5.20 & 5.30 & 5.34 & 5.22 & 5.30 \\
\hline $\mathbf{T}_{2}$ & 5.41 & 5.29 & 5.23 & 5.32 & 5.36 & 5.25 & 5.32 \\
\hline $\mathbf{T}_{3}$ & 5.42 & 5.30 & 5.34 & 5.34 & 5.39 & 5.28 & 5.35 \\
\hline $\mathbf{T}_{4}$ & 5.43 & 5.34 & 5.39 & 5.38 & 5.40 & 5.33 & 5.35 \\
\hline $\mathbf{T}_{5}$ & 5.50 & 5.44 & 5.41 & 5.45 & 5.48 & 5.37 & 5.42 \\
\hline $\mathbf{T}_{6}$ & 5.52 & 5.48 & 5.43 & 5.49 & 5.51 & 5.41 & 5.44 \\
\hline $\mathbf{T}_{7}$ & 5.45 & 5.37 & 5.40 & 5.40 & 5.42 & 5.35 & 5.37 \\
\hline $\mathbf{T}_{8}$ & 5.48 & 5.40 & 5.41 & 5.42 & 5.44 & 5.35 & 5.40 \\
\hline $\mathbf{T}_{9}$ & 5.55 & 5.52 & 5.50 & 5.54 & 5.56 & 5.45 & 5.50 \\
\hline $\mathbf{T}_{10}$ & 5.58 & 5.56 & 5.53 & 5.56 & 5.58 & 5.48 & 5.53 \\
\hline Mean & 5.47 & 5.40 & 5.38 & 5.42 & 5.45 & 5.35 & 5.40 \\
\hline SE (m) & 0.004 & 0.004 & 0.004 & 0.004 & 0.004 & 0.005 & 0.004 \\
\hline CD (5\%) & 0.011 & 0.011 & 0.013 & 0.012 & 0.012 & 0.014 & 0.012 \\
\hline
\end{tabular}


Fig.1 Effect of different seed treatments on vigour index - I during storage in wheat

\section{VIGOUR INDEX - I}

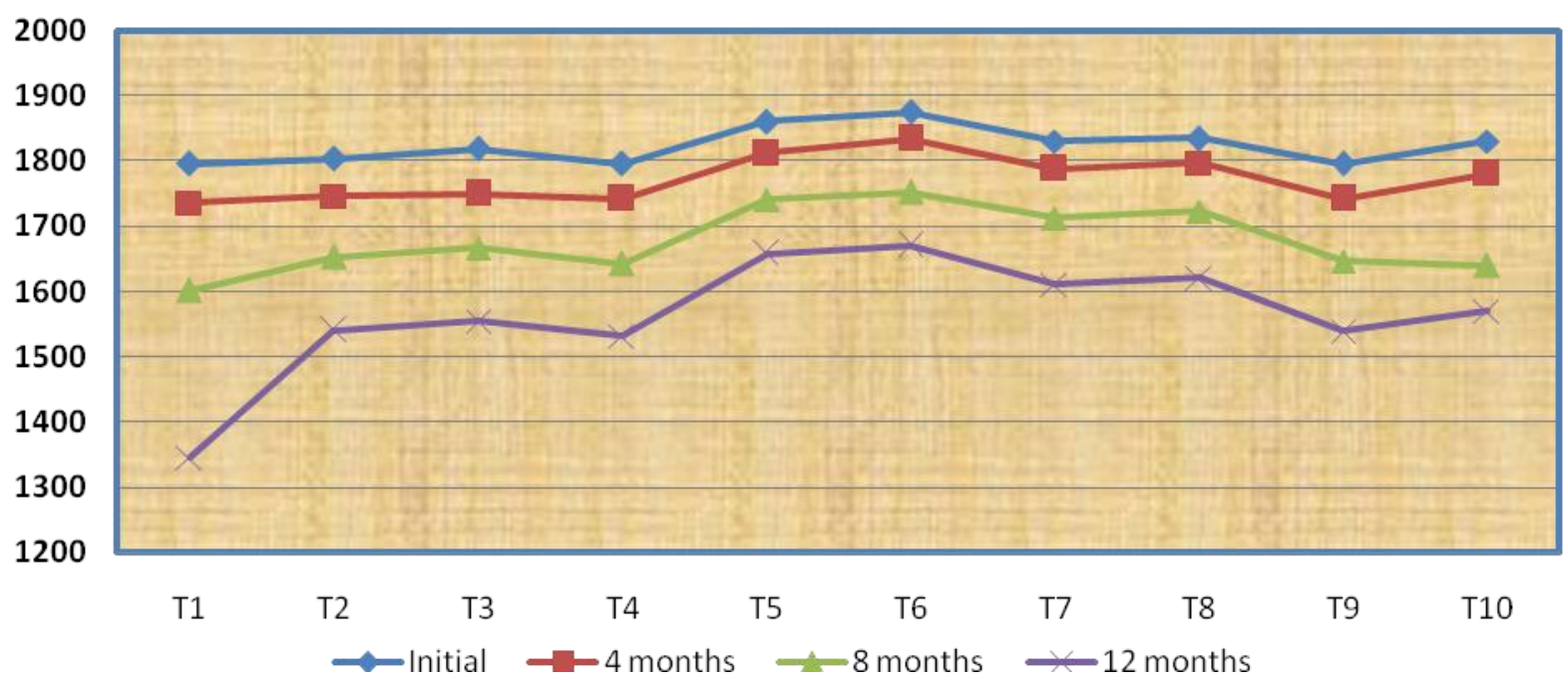

Fig.2 Effect of different seed treatments on vigour index - II during storage in wheat

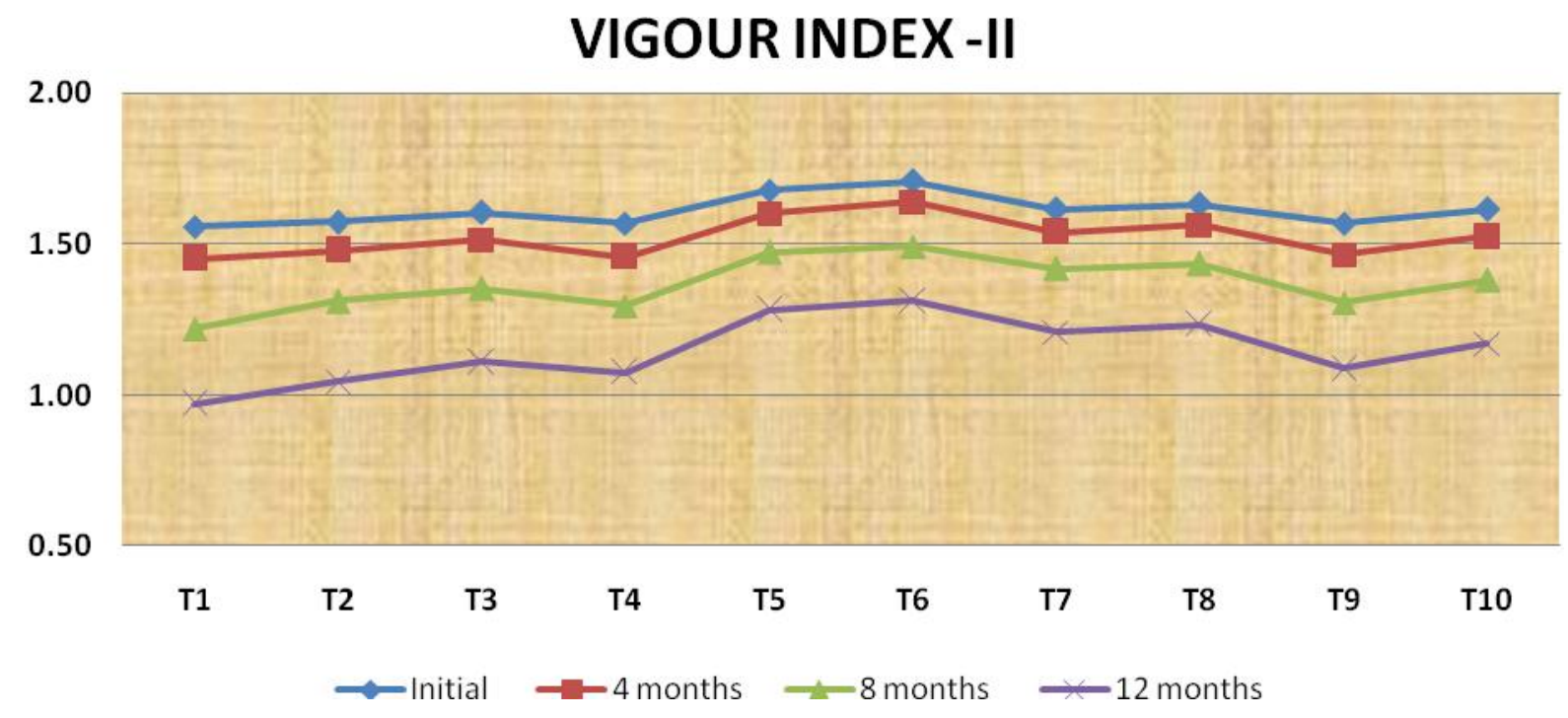

The computed vigour index, which is the totality of seed performance, has been regarded as a good index to measure the quality of seed lots. Irrespective of seed treatments, the vigour of the stored seed decreased with advancement in the storage period. Mean seedling vigour index - I and II recorded at the beginning and end of storage was 1824 to 1564 and 1.612 to 1.149 , respectively (Figure 1 and 2). The decrease in vigour index may be due to decline in germination, decrease in seedling length and seedling dry weight. In initial months of storage, significantly higher seedling vigour 
indices - I and II were recorded in treatment $\mathrm{T}_{6}-(1874$ and 1.707) which were at par with $\mathrm{T}_{5}$ - (1860 and 1.678), followed by $\mathrm{T}_{8}-(1834$ and1.633) which was at par with $\mathrm{T}_{7}-(1830$ and 1.615). The same pattern was recorded till six months of storage.

At $8^{\text {th }}$ month the trend varied and significantly highest vigour indices - I \& II were recorded in treatment $\mathrm{T}_{6}-(1753$ and 1.492) which was at par with $\mathrm{T}_{5}-(1741$ and 1.474), followed by $\mathrm{T}_{8}$ - (1723 and 1.434).

Same pattern was recorded till $12^{\text {th }}$ months of storage and significantly higher vigour indices - I and II were recorded in $\mathrm{T}_{6}$ - (1670 and 1.311) which was at par with $T_{5}-(1658$ and 1.283) as compared to $T_{1}$ - control (1346 and 0.970) after 12 months of storage. Higher vigour index in polymer + vitavax $200\left(\mathrm{~T}_{6}\right)$ may be due to more germination, seedling length and no infection and infestation by storage fungi and insects, respectively. The polymer treatment provides protection from the stress imposed by accelerated ageing. Similar results were reported by Sharma and Dhiman (2017) \& Parihar et al., (2019) in paddy and okra seeds. They reported that seeds treated with polymer and vitavax had resulted in higher seedling vigour Index-I as compared to untreated control after 12 months of storage.

The field emergence declined with advancing storage period due to different seed treatments. Mean field emergence recorded at the beginning and end of storage period was $92.36 \%$ and $82.70 \%$, respectively (Table 4 ).

Initially significantly highest field emergence (\%) was recorded for $\mathrm{T}_{6}-(94.33 \%)$ which was at par with $\mathrm{T}_{5}-(93.66 \%)$, followed by $\mathrm{T}_{8}$ - $(92.66 \%)$ which was at par with $\mathrm{T}_{7}$ (92.66\%), $\mathrm{T}_{10}-(92.66 \%), \mathrm{T}_{3}-(92.33 \%)$, followed by $\mathrm{T}_{2}-(91.33 \%), \mathrm{T}_{9}-(91.33 \%)$, and $\mathrm{T}_{4}-(91.33 \%)$ while the lowest was recorded in $\mathrm{T}_{1}$ - control $(91.33 \%)$. The same pattern was observed till six months
At the $8^{\text {th }}$ month the trend varied and significantly highest field emergence $(\%)$ was recorded for $\mathrm{T}_{6}-(89.33 \%)$, which was at par with $\mathrm{T}_{5}-(89.00 \%)$ followed by $\mathrm{T}_{8}-(88.66$ $\%), \mathrm{T}_{7}-(87.66 \%), \mathrm{T}_{10}-(87.33 \%), \mathrm{T}_{3}-(86.33$ $\%), \mathrm{T}_{2}-(85.33 \%), \mathrm{T}_{9}-(85.00 \%), \mathrm{T}_{4}-(84.66$ $\%)$ as compared to $\mathrm{T}_{1}-$ control $(80.33 \%)$. Same pattern was recorded till the end of storage period and significantly highest field emergence was recorded for $\mathrm{T}_{6}-(87.33 \%)$ which was at par with $\mathrm{T}_{5}-(86.66 \%)$ as compared to $\mathrm{T}_{1}$ - control $(76.00 \%)$. Seed treated with polymer and fungicides showed significantly higher field emergence over control. This could be due to protection of seeds by the chemicals and polymers against micro-organism, which in turn help in better establishment of seedling. The polymer and fungicide treatment keep seed intact, as it acts as binding material. It covers the minor cracks and aberration on the seed coat (Rao et al., 2017). The results recorded for field emergence are similar to the finding in radish (Kotia et al., 2020) where it was observed that radish seed treated with polymer @ $3 \mathrm{ml} / \mathrm{kg}+$ vitavax@2 g/kg resulted in significantly higher field emergence as compared to untreated control after 12 months of storage period.

The 100 seed weight (g) increased and decreased gradually during the storage period as per fluctuations in the prevalent temperature and humidity. On an average, 100 seed weight recorded at the beginning and the end of storage period was 5.47 and $5.40 \mathrm{~g}$, respectively (Table 5). Initially highest 100 seed weight was recorded for treatment $\mathrm{T}_{10}$ (5.58 g), followed by $\mathrm{T}_{9}-(5.55 \mathrm{~g}), \mathrm{T}_{6}-(5.52$ $\mathrm{g}), \mathrm{T}_{5}-(5.50 \mathrm{~g}), \mathrm{T}_{8}-(5.48 \mathrm{~g}), \mathrm{T}_{7}-(5.45 \mathrm{~g}), \mathrm{T}_{4}$ - (5.43 g), $\mathrm{T}_{3}-(5.42 \mathrm{~g})$ and $\mathrm{T}_{2}-(5.41 \mathrm{~g})$ over $\mathrm{T}_{1}$ - untreated control (5.40 g).

The similar pattern was recorded at the end of storage period. The highest 100 seed weight (g) was observed for $\mathrm{T}_{10}-(5.53 \mathrm{~g})$, followed 
by $\mathrm{T}_{9}-(5.50 \mathrm{~g})$ as compared to $\mathrm{T}_{1}$ - untreated control $(5.30 \mathrm{~g})$. Similar findings were reported in chickpea (Sushma 2013). The chickpea seeds treated with polymer @ 10 $\mathrm{ml} / \mathrm{kg}$ and Deltamethrin 2.8 EC @ $0.4 \mathrm{ml} / \mathrm{kg}$ of seed + Vitavax@ $2 \mathrm{~g} / \mathrm{kg}$ of seeds recorded significantly higher 100 seed weight as compared to untreated seeds after 4 months' storage period.

The study revealed that seed quality parameters declined with the advancement in storage period. The results have shown that seeds treated with polymer @ $3 \mathrm{ml} / \mathrm{kg}$ of seed + vitavax $200 @ 2 \mathrm{~g} / \mathrm{kg}$ of seeds $\left(\mathrm{T}_{6}\right)$ and vitavax $200 @ 2 \mathrm{~g} / \mathrm{kg}$ of seeds $\left(\mathrm{T}_{5}\right)$ recorded significantly superiority over control $\left(\mathrm{T}_{1}\right)$ for most of seed quality parameters during storage. Thus it can be concluded that for enhancing storability and to maintain the seed quality during storage, the seeds of wheat can either be treated with polymer coating@3 $\mathrm{ml} / \mathrm{kg}$ of seed + vitavax 200 @ $2 \mathrm{~g} / \mathrm{kg}$ of seed or vitavax $200 @ 2 \mathrm{~g} / \mathrm{kg}$ of seed.

\section{Acknowledgement}

The authors are thankful to the Department of Seed Science and Technology, CSKHPKV, Palampur for providing necessary laboratory facility and support for carrying out the study.

\section{References}

Abdul, B., and Anderson, J D. 1973. Vigour deterioration in soybean by multi criteria. Crop Science 13: 630-633

Anonymous. 1999. International rules for seed testing. Seed Science and Technology 27: 27-32

Doijode, S D. (2000). Seed viability and biochemical changes during storage of winter squash (Cucurbita maxima Duch.) seeds. Vegetable Science 27: 168-171

Duan, X, Burris. J S. 1997. Film coating impairs leaching of germination inhibitors in sugar beet seeds. Crop Science 37:515-520
Farooq, M., Wahid, A., Siddique, K H M. 2012. Micronutrient application through seed treatments- a review. J. Soil Science Plant Nutrition 12: 125-142

Geetha, V V., and Bhaskaran, M. 2020. Standardization of suitable drying methods for storing groundnut and sesame seeds. International Journal of Current Microbiology and Applied Sciences 9: 478-485

Gevrek, M N., Atasoy, G D., Yigi, A. 2012. Growth and yield response of rice (Oryza sativa) to different seed coating agents. International Journal Agriculture and Biology 14: 826-830.

Guan, Y., Wang, J., Tian, Y., Hu, W., Zhu, L. 2013. The novel approach to enhance seed security: dual anti-counterfeiting methods applied on tobacco pelleted seeds. PLoS One 8: 210-219

Huang, P., He, L., Abbas, A., Hussain, S., Du, D., Hafeez, MB., Balooch, S., Zahra, N., Ren, X, Rafiq, M., and Saqib, M. Seed priming with Sorghum water extract improves the performance of Camelia (Camelia sativa (L.) Crantz.) under Salt Stress. Plants 10: 749-764

Jacob, R S., Kumar, M B., Varghese, E and Sinha, S N. 2016. Hydrophillic polymer film coat as a micro-container of individual seed facilitates safe storage of tomato seeds. Scientia Horticulturae 204:116-122

Johnson, G A., Hicks, D H., Stewart, R F and Duan X. 1999. Use of temperatureresponsive polymer seed coating to control seed germination. Acta Hortic. (ISHS) 504: 229-236

Kotia, K., Dhiman, K C and Kanwar R. 2020. Effect of seed treatments on quality and storability of radish (Raphanus sativus L.) seeds. Agriculture research journal 57: 414-424

Kumar, V., 2007. Effect of seed coating with polymer, fungicide and insecticide on seed quality in cotton during storage. Karnataka Journal of Agricultural Science 20: 137139

Mastouri, F., Björkman, T., Harman, GE. 2010. 
Seed treatment with Trichoderma harzianum alleviates biotic abiotic, and physiological stresses in germinating seeds and seedlings. Phytopathology 100: 12131221

Lagoa, A O., Ferreira, A C., Vieira, R D. 2012. Plantability and moisture content of naked and pelleted seeds of supersweet (Sh2) corn during cold storage conditions. Revista Brasileria de Sementes 34:39-46

Padhi, S K., Behera, S., Mishra, S P., Padhiary, A K and Nayak B. 2017. Effect of seed coating materials on seed quality during storage of paddy. Journal of Pharmacognosy and Phytochemistry 6: 1263-1279

Parihar, P., Dhiman, K C., Kapila, R K and Kanwar R. 2019. Effect of coating on quality of okra seeds in storage. Seed research 47: 36-43

Rao, P S., Rani, M S., Ankaiah, R., Rajasri, M and Kumari M. Effect of Seed coating with polymer, fungicide and insecticide on seed quality in sorghum (Sorghum bicolor (L.) Moench) during storage. International Journal of Bio-resource and Stress Management 8:226-230

Roopashree, B., Joll, R B., Ashok, S S and Motagi B N. 2018. Effect of Polymer coating and fungicide on storage performance of kabuli chickpea varieties. International Journal of Current Microbiology and applied sciences 7: 954961

Sushma, P P. 2013. Effect of polymercoat and seed treatment chemicals on seed storability and field performance of chickpea. M.Sc. Thesis, Department of Seed Science and Technology, University of Agricultural Sciences, Dharwad
Sheoran, O P., Tonk, D S., Kaushik, L S., Hasija, R C and Pannu R S. 1998. Statistical Software Package for Agricultural Research Workers. Recent Advances in information theory, Statistics \& Computer Applications by D S Hooda \& R C Hasijia, Department of Mathematics Statistics, CCS HAU, Hisar pp 139-143

Sharratt, B S., Gesch, R W. 2008. Emergence of polymer-coated corn and soybean influenced by tillage and sowing date. Agronomy Journal 100:585-590.

Sherry, R J., Sinha, S N., Kumar, M B A, and Srivastava C. 2007. Enhancement of efficacy of seed protectant through polymer seed coating in tomato. PUSA AgriScience 30: 42-46.

Sharma, J., Dhiman, K C., Sharma, J K and Kumar R. 2017. Effect of seed coating on seed yield and related parameters in quality protein maize hybrid HQPM 1. Himachal Journal of Agricultural Research 43: 68-72

Sharma, A., and Dhiman, K C. 2017. Effect of seed coating with synthetic polymer and chemicals on seed quality and storability of rice (Oryza sativa L.). Himachal Journal of Agricultural Research 43:102111

Vanangamudi, K., Srimathi, P., Natarajan, N., Bhaskaran, M. Current scenario of seed coating polymer. ICAR: Short Course on Seed Hardening and Pelleting Technologies for Rainfed or Garden Land Ecosystems on maize, 2003, 80-100.

Ziani, K., Ursua, B, and Mate J I. 2010. Application of bioactive coatings based on chitosan for artichoke seed protection. Crop Protection 29: 853-859.

\section{How to cite this article:}

Anubhav Thakur, K. C. Dhiman and Rajesh Kanwar. 2021. Effect of Seed Treatment on Seed Quality and Storability in Wheat (Triticum aestivum L.) in North Western Himalayas. Int.J.Curr.Microbiol.App.Sci. 10(08): 565-577. doi: https://doi.org/10.20546/ijcmas.2021.1008.067 